\title{
THE COURSE OF THE GENICULO-CALCARINE VISUAL PATH IN RELATION TO THE TEMPORAL LOBE
}

BY

\author{
H. M. Traguair, M.D. \\ EDINBURGH
}

ClEar and exact accounts of the anatomical course of the visual fibres between the external geniculate body and the calcarine cortex are lacking in most text books whether of anatomy, ophthalmology or neurology; as a rule it is merely stated that these fibres pass backwards around the posterior horn of the lateral ventricle into the occipital lobe forming the optic radiation. A detailed description is given by Henschen ${ }^{(1)}$ :-From the external geniculate body the visual fibres pass outwards, then downwards, parallel with the lateral wall of the posterior ventricular horn where they form a compact bundle 5 to $10 \mathrm{~mm}$. in vertical measurement. Then turning backwards they pass to the occipital lobe in a horizontal direction about the level of the second temporal convolution and second temporal sulcus. A section 6 to $7 \mathrm{~cm}$. in front of the occipital pole shows the visual path as an enclosed bundle about 5 to $10 \mathrm{~mm}$. in height and 2 to $3 \mathrm{~mm}$. thick at the latero-ventral angle of the posterior horn. As the fibres pass backwards they approach the ventricle and spread out on its ventral aspect. In this bundle the dorsal retinal quadrants are represented by the dorsal, and the ventral retinal quadrants by the ventral fibres. Henschen points out that the term "optic radiation " (Sehstralung) is often too loosely used as if it referred to the whole of the sagittal marrow of the temporal, parietal and occipital lobes, whereas it is only the outer stratum of the ventral part of this formation which conveys visual impulses and therefore constitutes the visual path. In this pathway homonymous fibres lie together.

Another clear description, differing in important details from that of Henschen, is given by Meyer (2, 3), who regards the geniculo-calcarine visual path as consisting of three segments, dorsal, lateral, and ventral. Only the dorso-lateral fasciculi pass directly to the occipital cortex, the ventral fibres pass downwards and forwards into the uncinate region of the temporal lobe, making a long detour or " temporal loop" around the anterior horn of the lateral ventricle. The most ventral fibres pass farthest forwards and extend practically to the tip of the ventricular horn, around which they turn downwards and then backwards beneath the ventricle to the most anterior part of the inferior calcarine cortex. Meyer points out that lesions of the anterior part of the temporal lobe, if sufficiently deep, involve the most ventral bundle of the 
optic radiation and therefore imply a defect in the upper quadrant of the visual field. In a rècent paper by Cushing ${ }^{(4)}$ this arrangement of the geniculo-calcarine fibres has been illustrated by drawings made from glass model reconstructions prepared by Meyer himself, and the accompanying figure represents diagrammatically the temporal detour. It will be seen that, according to this view, the visual fibres, on leaving the external geniculate body, do not form a compact bundle but spread out widely, especially in the vertical plane, while the presence of the ventral fasciculus so far forwards in the temporal lobe would obviously be of great importance in connection with the diagnosis of temporal lobelesions since

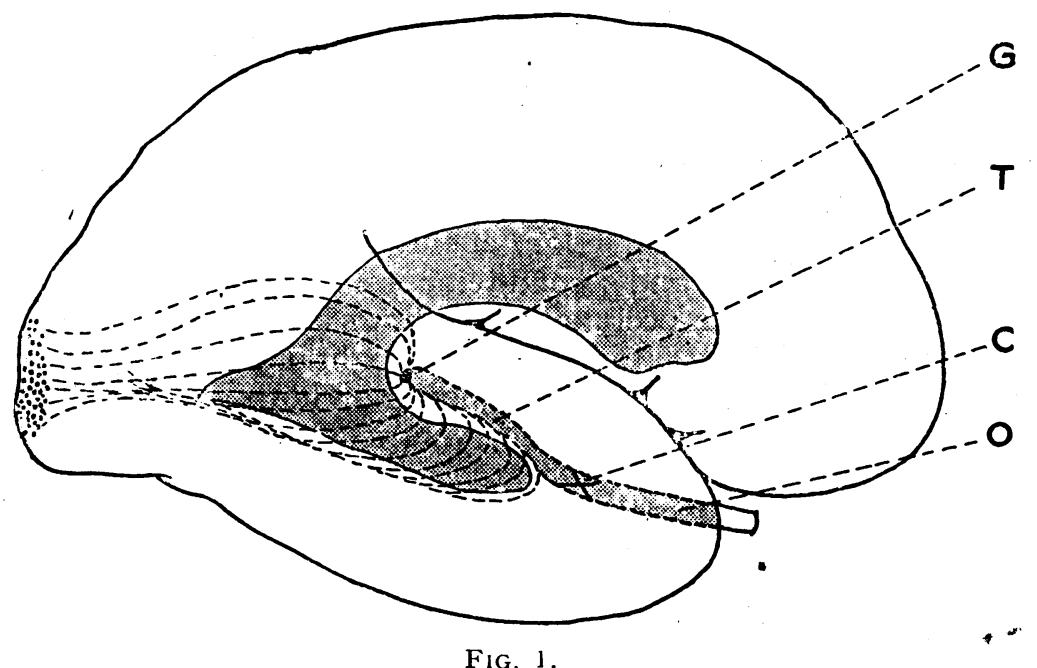

Diagrammatic representation of the geniculo-calcarine pathway according to Meyer Modified from Cushing (loc. cit).

G: geniculate body. T. Optic tract. C. chiasma. O. optic nerve.

interference at this part would produce homonymous hemianopsia varying in extent and intensity according to the nature of the lesion.

"Cushing's paper deals with the perimetric evidence of temporal lobe lesions from the point of view of the field defects produced by interference with the geniculo-calcarine fibres, especially in their course through the temporal lobe. The first case $^{(5)}$ which drew attention to the subject was one observed in 1910, in which fits with symptoms indicative of uncinate origin were present. Some years previously the patient had been shot in the left eye, and the bullet was shown by a radiogram to be lodged in the petrous portion of the left temporal bone, having evidently traversed the lower part of the left temporal lobe. The field of vision of the 
right eye showed no gross change, but Professor Meyer, happening to see the case, suggested that a few of the ventral visual fibres might have been damaged, and that a closer examination might elicit some defect. Re-examination demonstrated a narrow sector defect in the upper outer quadrant just outside the vertical meridian, extending downwards towards the central area, i.e., in the position in which a limited lesion of the ventral crossed fibres of the right eye would naturally produce a field change. The presumption followed that had the left eye been present its field would have shown a corresponding homonymous alteration. This case will be referred to again later.

In the present series, out of 276 verified supratentorial tumours, 59 affected the temporal lobe. In 39 of these it was possible to examine the visual fields, and homonymous field defects indicating involvement of the temporal loop of the optic radiation were present in thirty-three instances. The six exceptions deserve careful consideration. Three were slow-growing endotheliomata, and one an angioma deforming the lobe from without. One was a dermoid cyst in the tip of the lobe associated with uncinate seizures. The sixth was a glioma in which, however, the perimetric evidence obtained was not regarded as complete.

In the thirty-three cases with demonstrated homonymous field defects the hemianopsia was complete in eight at the time of the first examination. None of these showed recovery of the field after operation, and it is suggested that in many or all of them the geniculate body may have been involved or the optic tract affected by pressure. In four of these eight cases the fixation point was bisected, in four it was spared during the time the cases were under observation.

In the remaining twenty-five cases partial homonymous hemianopsia of quadrantic type was present. These field defects fell into three classes : (1) stationary ; (2) advancing towards complete hemianopsia; and (3) receding after successful operation.

Ten cases have been selected for the purpose of this paper, all belonging to the group of partial homonymous hemianopsias, and recorded with photographs and charts of the visual fields. The field changes exhibit characteristic and interesting features of which four stand out prominently.

(1.) The edge of the defect is sloping, i.e., the transition from the seeing field to the blind area is gradual across a partially amblyopic zone, the grade varying in steepness in different cases and in different parts of the same field.

(2.) The defect is usually more marked on the side of the lesion, i.e., in the homolateral nasal field than in the heterolateral temporal field (eight out of ten). The cases thus exhibit incongruity in homonymous hemianopic fields in a pronounced degree. 
(3.) Where the lesion is low the field defect is high and vice versa.

(4.) Recovery after successful operation is frequent.

Somewhat similar fields are illustrated from cases of fracture of the base of the skull and temporal lobe abscess.

This' clinical evidence has a double interest in regard to two distinct though associated questions : firstly, in connection with the diagnosis of temporal lobe lesions; secondly, in relation to the course of the geniculo-calcarine visual path.

The bearing of these field changes on the diagnosis of temporal lobe lesions and particularly of tumours is fully dealt with in Professor Cushing's paper, and it is not the object of this review to discuss this aspect of their importance. Cushing and his assistants have brilliantly demonstrated the value of perimetry, especially of quantitative perimetry, in the localization of tumours in what has hitherto been regarded as one of the most silent areas of the brain. Although homonymous hemianopsia has long been recognized as a frequent accompaniment of temporal lobe lesions, both tumour and abscess, Cushing's study is the first in which this symptom has been adequately investigated by modern perimetric methods, and in which its frequency has been estimated and its indications pointed out.

The second question is:-What is the anatomical significance of these field changes; in other words, do they support the view that the geniculo-calcarine visual fibres form a loop in the temporal lobe as described by Meyer? Throughout the paper it is assumed that the hemianopsia is due to involvement of the optic radiation in the temporal lobe, although, as already mentioned, it is pointed out that in the later stages the geniculate body and the tract may be affected. The possibility of some other site of interference is not dealt with, and it is somewhat surprising that, except by one speaker (Spiller), this question was not referred to in the discussion when the paper was read at the American Neurological Association.

If we consider the first case quoted, in which a bullet injury of the lower part of the left temporal lobe was associated with a right upper outer field defect, it seems desirable to consider alternative possibilities before this result can be regarded as evidence of the presence of the visual path in that situation. There was a fracture of the base, possibly with some radiation, with haemorrhage and subsequent cicatrisation. Under these circumstances it is not at all improbable that the tract or chiasma was affected, and it is certainly difficult to exclude this explanation. The notch in the right field might equally well have represented one side of a bitemporal hemianopsia or of a homonymous hemianopsia of tract origin. 
$\mathrm{Up}$ to the present, homonymous hemianopsia in connection with temporal lobe lesions has been attributed to tract interference or, if the lesion is deep enough, to interference with the external geniculate body or the anterior part of the optic radiation in its hitherto accepted position. In the absence of other guiding signs an indication as to the site of the visual block must be sought from an examination of the character of the field changes, which, in the present instance, seems to call for some explanation, and is too important to be neglected. The chief features of the defects have already been referred to. Of these the sloping edge, the relation of the position of the lesion to that of the defect, and the tendency to recovery after operation are all compatible with a suprageniculate site of interference. On the other hand, the most prominent and most significant feature, the incongruity, speaks strongly for subgeniculate, i.e., tract interference.

Incongruity in homonymous hemianopic fields has been the subject of considerable attention and thought amongst ophthalmologists. It is discussed by . Wilbrand ${ }^{(7)}$, and has been ably reviewed by Roenne( ${ }^{(8)}$, who points out that its localizing value was first drawn attention to by Bjerrum in 1892. Its significance was also independently appreciated by Schirmer ${ }^{(9)}$ : Of the various causes of incongruity there is only one which could produce it so consistently in a series of cases as in those under review, and that is the incidence of the interference at a point in the visual pathway where fibres from corresponding retinal points-or minute retinal areas-have not yet come to lie together. We must, therefore, believe that in temporal lobe lesions the visual block takes place below the geniculate body, or, that in the optic radiation, at least in its anterior part, fibres from corresponding retinal points do not lie in juxtaposition.

The point at which the homologous fibres come together is not known with certainty; Roenne believes that the rearrangement is frequently completed in the lower part of the tract, while according to Henschen, "as far as we know," it takes place at the geniculate body. No observer has hitherto suggested that the separation persists above this level, though Roenne concludes, rather reluctantly, that small irregularities of arrangement may exist in individual cases, affecting, however, only a narrow zone along the vertical meridian of the field. If the fields depicted are to be regarded as evidence that the visual pathway is affected in the temporal lobe they must also be held to show that the homologous retinal fibres are separate at this level. In this case, since the uncrossed fibres are so frequently first and more severely affected, it must be supposed that the crossed fibres occupy a situation relatively less exposed to interference by tumours in their usual position in the substance of the lobe; and that the cases with early 
contralateral field changes are associated with tumours in different and less common sites. Evidence on this point would be of interest.

If homologous retinal fibres do not lie together in the anterior part of the optic radiation they must become associated at some more posterior point, for clinical evidence indicates that they do come together. On this reasoning the more posterior the interference the less the incongruity should be in the fields. Again, if the most ventral fibres extend most anteriorly in the temporal loop in the way shown in the diagrams, the most anterior tumours whether high or low should produce field defects limited to the periphery of the upper field quadrants. Whether these relationships occur frequently can hardly be estimated from the ten cases reported, but in any case they would be capable of another explanation. It is the anterior part of the temporal lobe which lies most directly beneath the tract, in which homologous fibres lie more or less separate, and a swelling of the posterior part of the lobe would be more likely to affect the termination of the tract or even the geniculate body and the beginning of the radiation, in which homologous fibres are believed to lie together.

Other interesting questions arise in this connection which, however, cannot be usefully discussed in the absence of details of a larger number of cases.

A very striking feature of the fields is the close resemblance they show to those of tract hemianopsia due to pituitary tumour, our knowledge of which we chiefly owe also to Walker and Cushing ${ }^{(10)}$. Here the crossed fibres are usually affected first, so that the contralateral field shows the greater defect, but, as these authors have shown, it may be otherwise. The only difference in the field changes is the rather greater frequency of central scotoma in the pituitary cases. The present writer believes that central scotoma in such cases indicates the acuity of pressure rather than the site, and depends upon the impairment of the vascular supply to the macular bundle. Thus, in chronic acromegalies with very slowly growing tumours, central scotoma is usually absent, while with moderately rapid or aggressive growth this feature is more prominent, and especially so in inflammatory conditions. No significance, then, as to the site of interference can be attached to the absence of central scotoma in a case of slowly growing temporal lobe tumour. In this connection it may be noted that in the two cases of traumatic homonymous hemianopsia recorded the defects are scotomatous in type.

The presence of papilloedema shows that the optic nerve sheath is open, and is, therefore, against pituitary tumour, but it does not otherwise indicate the site of interference. The hemianopic pupillary reaction is usually absent or indefinite in relative hemianopsias, but 
it is of interest to note that it is present in complete hemianopsia in temporal lobe cases, showing clearly that the tract does become affected. Post-mortem records show that compression of the tracts has been frequently found in cases of temporal lobe tumour (Willbrand and Saenger ${ }^{(7)}$ ), and in the early stages of this process relative hemianopsia with incongruity would be produced. There appears to be no reason to suppose that compression of the tract occurs only in the more advanced stages of enlargement of the temporal lobe, especially when we remember how closely the uncus and hippocampal gyrus are applied to its lower outer side, the part in which the uncrossed fibres are contained. The recorded charts show that incongruity may be pronounced in early cases. In the case of a sufficiently large growth both sub- and suprageniculate interference might, of course, be present, and it would be impossible to separate the symptoms by perimetry.

As minor points we may note the recovery of the fields after operation as indicating that the visual path was compressed, but not invaded by the tumour, and also that the operative measures produced no permanent damage, features which seem to favour tract compression, though not necessarily incompatible with suprageniculate interference by an encapsulated growth; and also the absence of hemianopsia in six cases, mostly slowly advancing, which falls into line with what we know of the reaction of the chiasma and tracts to slowly growing pituitary tumours. The extreme restriction of the field which was present in several cases on the side of the lesion may be explained by oedema of the optic nerves of some duration, which also causes the peripheral restriction-in reality general depression-of the opposite field whether hemianopic or not. The term "tubular" is only applicable to fields whose linear diameter remains the same for the same visual angle at any distance. Such fields are necessarily always of psychical origin.

An interesting feature is present in the fields of two of the recorded cases, Nos. 6 and 10 . In the first, a left hemianopsia, the fixation point in the left field is included in the defective half field, like a reversed sparing of the macula, central vision being $20 / 50$, while the right eye with bisected fixation point had V. $=20 / 20$. There was severe papilloedema and a rapidly growing and ultimately extensive right-sided glioma. In the second case, a right superior quadrant hemianopsia due to a meningeal endothelioma of the left temporal fossa, the field of the left eye showed considerable general restriction and an arcuate defect* of

* Arcuate defects in papilloedema are rare. Roenne has seen several cases and ascribes the defect to an accidentally specially severe implication of a fibre bundle at the disc margin. (Arch. f. Augenheilk., Vol. LXVI, Part 3-4, p. 191, 1913. Von Szily describes a case in which this was probably a complication (Klin. Monatsbl. $f$. Augenheilk, Vol. LI, p. 196, February, 1913). 
the nerve fibre bundle of Bjerrum type curving from the blind spot below the fixation point and up into the upper nasal quadrant which was deeply indented. V. $=10 / 50$. The right field showed a very slight upper outer notch. V. $=10 / 10$. Bilateral papilloedema. Thus, in these two cases the hemianopsia was impure.

In the first case either the central uncrossed fibres of the left eye had become affected-an explanation which seems unlikely-or, more probably, the central failure was due to a macular complication depending on the papilloedema. In the second, however, the curved defect crossing the vertical meridian provides a sure indication of involvement of the optic nerve, and this, together with the reduction in central vision and the peripheral restriction of the temporal field, suggests that the site, or at least $a$ site, of interference was at or near the chiasmal termination of the left optic nerve.

Looking at the question from the opposite point of view, we can find no evidence against tract interference and in favour of supra-geniculate involvement of the visual fibres in the lower and anterior part of the temporal lobe. Congruity of the defects, in time, extent, and intensity, would be required, or evidence of homonymous hemianopsia, in a lesion of the anterior part of the temporal lobe under conditions which would exclude the probability of sub-geniculate interference. Unfortunately such a lesion must be rare in clinical experience.

The solution of the problem rests on the correct interpretation of perimetric evidence based on the anatomy-in the widest sense -of the visual pathway and on the mechanism of production of field changes by tumour pressure. The essential factor in this process is, in the present belief of the writer, vascular nutritional impairment. It is this which enables a rounded surface like that of a pituitary tumour or the inner side of the temporal lobe, by producing a local ischaemia at the point of pressure incidence on the chiasma or tract, to give rise to a field defect with sloping edges which recovers with great rapidity if the pressure can be relieved before actual atrophy has resulted. This explanation is offered with full appreciation of the difficulty of proof, but some help may be obtained from the clinical study of certain tumours and other lesions affecting the spinal cord.

As regards the temporal lobe it is to be hoped that more records will be published with minute details, so that the exact position of the lesion may be compared with the field changes ascertained by careful quantitative perimetry in a large number of cases. The value of this method in the diagnosis of temporal lobe lesions has been fully established by Professor Cushing. As regards the site 
of interference the perimetric evidence at present available indicates the optic tract rather than a temporal detour of the geniculo-calcarine fibres.

\section{REFERENCES}

1. Henschen.- " Handbuch der Neurologie." Lewandowsky, Berlin, 1910, Vol. I, p. 903-914, Vol. III, p. 773 et seq.

2. Meyer, A. - The connections of the Occipital Lobes and the present status of the Cerebral Visual Affections." Trans. of the Assoc. of Amer. Physicians, 1907, Vol. XXII, p. 7.

3. Idem.-American Neurological Association, 1911.

4. Cushing.- "The Field Defects produced by Temporal Lobe Lesions." Trans. Amer. Neurol. Soc., 1921, and Brain, Vol. XLIV, p. 341. 1921.

5. Cushing and Heuer.- "Distortions of the Visual Fields in Cases of Brain Tumour." Johns Hopkins Hosp. Bulletin, June, 1911, Vol. XXII, p, 190.

6. Discussion on Professor Cushing's paper on "The Field Defects of Temporal Lobe Lesions." Trans. Amer. Neurol. Assoc., June, 1921.

7. Wilbrand and Saenger.- "Die Neurologie des Auges," 1917, Vol. VII, p. 152, et seq.

8. Roenne. - "Ueber die Inkongruenz und Asymmetrie im homonym hemianopischen Gesichtsfeld." Klin. Monatsbl.f. Augenheilk., 1915, Vol. LIV, p. 399.

9. Schirmer.-"A Case of Incongruous Homonymous Hemianopsia." "Arch. of Ophthal., 1912, No. 2, Vol. XLI, p. 136.

10. Walker and Cushing.- "Chiasmal Lesions with especial reference to Homonymous Hemianopsia with Hypophyseal Tumour." Arch. of Ophthal., 1918, No. 2, Vol. XLVII, p. 124.

\section{A CASE OF QUININE AMBLYOPIA BY}
H. GAINSBOROUGH,
AND
R. GainsBOROUGH,
M.B., B.Ch.Cantab.
L.R.C.P., M.R.C.S.

MEDICAL REGISTRAR, ST. GEORGE'S HOSPITAL

A. C., age 41, a chauffeur, was discharged from his employment. At 3 a.m. one morning soon after, while under the influence of drink, he swallowed the remaining contents of a bottle of 5 grain quinine tablets, taking probably about 60 grains. He thought he had a malarial relapse, but he had not had such for a long time, and this was the first dose of quinine he had taken for three years. Possibly there was suicidal intent. He fell asleep again, but a few hours later awakened, feeling considerable distress and giddiness, and within three hours he had lost his sight. $\mathrm{He}$ had considerable nausea and vomited slightly, but there were no subjective auditory sensations.

On examination by one of us (R. G.) eight hours after he had taken the drug, he was found lying in bed; the respiration was quick and shallow, but his colour fairly good; the pulse was rapid, thready and irregular, and the rate was 120 . His sight was gone, he being only just able to distinguish light from dark, but not being able to point to the direction of the light. The pupils were fixed and dilated. The fundi were pale and hazy, the arteries much 